\title{
Relating aspects of critical nutrition literacy at the personal level: using structural equation modelling to empirically test hypotheses
}

Desire Alice Naigaga ( $\nabla$ dnaiga@oslomet.no)

OsloMet - Oslo Metropolitan University https://orcid.org/0000-0003-2223-2112

Kjell Sverre Pettersen

OsloMet - storbyuniversitetet Kjeller Campus

Sigrun Henjum

OsloMet - storbyuniversitetet Kjeller Campus

Øystein Guttersrud

Norwegian Centre for Science Education

\section{Research}

Keywords: Adolescents, Critical nutrition literacy, Self-efficacy, Rasch modelling, Structural equation modelling

Posted Date: December 11th, 2019

DOI: https://doi.org/10.21203/rs.2.18579/v1

License: (c) (i) This work is licensed under a Creative Commons Attribution 4.0 International License. Read Full License 
1 Relating aspects of critical nutrition literacy at the personal level: using 2 structural equation modelling to empirically test hypotheses

3

4 Author details

5 Desire Alice Naigaga ${ }^{1}$, Prof. Kjell Sverre Pettersen ${ }^{1}$, Prof. Sigrun Henjum ${ }^{1}$, Øystein 6 Guttersrud $^{2}$

\section{$7 \quad$ Institutional affiliations}

$8 \quad{ }^{1}$ Department of Nursing and Health Promotion, Faculty of Health Sciences, OsloMet - Oslo 9 Metropolitan University, Kjeller, Norway.

$10{ }^{2}$ Norwegian Centre for Science Education, University of Oslo, Blindern, Norway.

11 Corresponding author

12 Email: desirenaigaga@gmail.com 


\section{Research snapshot:}

2 Study hypothesis: adolescents' critical nutrition literacy at personal level is related to their 3 self-efficacy in the science topic 'Body and Health'.

4

$5 \quad$ Key findings: The sample data strengthened the specified structural equation model. 6

7 Keywords: Adolescents, Critical nutrition literacy, Self-efficacy, Rasch modelling, Structural 8 equation modelling 


\section{Abstract}

2 Background: Whereas the majority of efforts targeting adolescents' dietary behaviour have predominantly focused on improving their access to nutrition information, findings indicate that this approach has had little if any effect on their short-term dietary behaviour. Among the reasons for this is that adolescents report finding it difficult to understand the nutrition information that they access, and are thus unable to use the information as intended. Exploring critical nutrition literacy (CNL) might provide insight into how adolescents evaluate and apply the nutrition information that they get from different sources. Methods: The present study employed a cross-sectional study design, in which we sampled 1620 tenth graders, aged 15 and 16 years, enrolled at 58 secondary schools in Norway. Data collection: Using an electronic survey system, the participants responded to scales measuring 'selfefficacy in Body and Health' (SEBH), 'evaluation of nutrition information' (CNLEval and 'engagement in dietary behaviour' (CNLEng). Study hypotheses: We hypothesized that SEBH influences adolescents' CNLEval and CNLEng, and that CNLEval influences CNLEng directly and mediates the effect of SEBH on CNLEng. Statistical analyses: Using Lisrel 9.30, the present study formulated and evaluated a hypothesized structural equation model (SEM) linking the two subdomains of CNL (CNLEval, CNLEng) and SEBH. Results: The study yielded a simple yet theoretically sound model linking CNL and SEBH; the adequate fit to the goodness-of-fit indices showed that the proposed model adequately described the data. Conclusions: The present study strengthened the hypothesis that there exists a strong association between CNL and SEBH, and between the two aspects of CNLCNLEval and CNLEng. Hence, efforts aimed at promoting positive dietary behaviour in adolescents and enhancing their nutrition literacy efforts might benefit from enhancing their perceived self-efficacy in nutrition related subjects. 
2 Health literacy is context-specific, taking different forms within the field of health, and bears different meanings to different people [1]. One important domain of HL is nutrition literacy (NL), which could be defined as 'the capacity to obtain, process and understand nutrition information needed to make appropriate decisions regarding one's health' [2].

Nutbeam's tripartite model of HL classifies NL into the three levels functional nutrition literacy (FNL), interactive nutrition literacy (INL) and critical nutrition literacy (CNL) [3-5]. FNL refers to the basic writing and reading skills that are required to access information about nutrition, while INL is comprised of the interpersonal communication and cognitive skills which enable individuals to translate and apply information in their daily lives with the aim of improving their overall nutritional status. Thirdly, CNL refers to proficiency in critically analysing nutrition information and advice, alongside increased awareness and engaging in emancipatory action to address barriers to good nutrition at personal, social and global levels [4-6]. Furthermore, at the individual level, CNL might be assessed by its two aspects, 'critical evaluation of nutrition information' (CNLEval) and 'engagement in dietary behaviour' (CNLEng) [7].

During adolescence individuals develop their dietary behaviours [8], and it is therefore plausible that improving NL during adolescence might increase their chances of developing healthy dietary behaviours and prevent health risks during adulthood.

Studies show that adolescents generally find nutrition information difficult to understand [9]; they inadequately interpret nutrition information [10] and are unable to establish the credibility of the sources of this information [11]. Therefore, it is not surprising that in spite of the increased awareness and access to nutrition information, adolescents do not always use this information properly when making dietary choices [12-13]. This might suggest that in order to improve the NL of adolescents, it is important to provide nutrition information that they can easily understand. By exploring the 'critical' dimension of nutrition literacy, stakeholders might be better informed about how adolescents understand nutrition information, what cues they use to interpret the information, and can therefore tailor the information accordingly.

\section{Measuring CNL in adolescents:}


1 At present, there are only a few instruments for assessing NL, and even fewer for assessing

2 CNL [9, 14]. The bulk of existing CNL instruments have mainly been developed and 3 validated using classical test theory (CTT) techniques; while CTT has long-standing benefits,

4 it yields instruments that are sample-dependent and therefore require repeated validation prior

5 to use on different samples; this is costly and limits the use of existing instruments. For this

6 reason, researchers are adopting the use of modern measurement approaches such as item

7 response theory (IRT) that have the advantage of being sample-test independent, and as a

8 result, yield psychometrically defendable scales.

Materials and methods:

The present study applied three Rasch-validated scales, two CNL scales (CNLEval, CNLEng, and one measuring self-efficacy (SEBH) on an adolescent sample. Applying IRT approaches to validate measures of NL, makes it possible to identify bias at item level, yielding more psychometrically robust measures of NL across different populations [15]. The use of Rasch modelling for scale validation in the field of NL is discussed in detail elsewhere [6. 7. 16]. The present study applied the five-item CNLEval scale as a measure of the adolescents' perceived proficiency in evaluating nutrition information from various sources (Table 1). The prior validated scale uses a six-point rating scale and captures skills required for evaluating the 'consistency' and 'trustworthiness' of nutrition information [16].

\section{Table 1: Stem and wording of the items in the CNLEv scale ${ }^{a}$}

"Nutrition" refers to the connection between diet and health. On a scale from "very difficult" to "very easy", how easy or difficult would you say it is to $(1=$ Very difficult, $6=$ Very easy)

\begin{tabular}{|l|l|}
\hline Item & Item wording \\
\hline 1 & $\begin{array}{l}\text { evaluate whether nutritional advice in the media (newspapers, magazines, } \\
\text { television) is reliable? }\end{array}$ \\
\hline 2 & $\begin{array}{l}\text { consider how reliable warnings about poor nutrition are, as warnings against } \\
\text { malnutrition? }\end{array}$ \\
\hline 3 & $\begin{array}{l}\text { consider whether information on websites for nutritional information is } \\
\text { reliable? }\end{array}$ \\
\hline 4 & \begin{tabular}{l} 
consider what it takes a scientific nutritional claim to be valid? \\
\hline
\end{tabular}
\end{tabular}


${ }^{a}$ Items originally stated in Norwegian.

\section{Table 2 Stem and wording of the items in the CNLEng scale ${ }^{a}$}

How do you agree with the claim ( 1 = Disagree strongly, 6 = Agree strongly $)$ ?

\begin{tabular}{|l|l|}
\hline Item & Item wording \\
\hline 1 & $\begin{array}{l}\text { I am concerned about eating foods that provide the nutrients my body needs } \\
\text { I am concerned that there are healthy foods in the grocery shops that my } \\
\text { family shops at }\end{array}$ \\
\hline
\end{tabular}

${ }^{a}$ Items originally stated in Norwegian.

Adolescents' engagement in dietary behavior in the present study was measured using the 'personal' aspect from the prior evaluated 'Engagement in dietary behaviour (EDB) scale' that measures adolescents' engagement in dietary behaviour at the personal, social and global levels [7]. This aspect is measured using two items related to how concerned the adolescents are about eating healthy foods and having a variety of healthy foods available to them (Table 2).

Whereas it is inarguably important, the link between NL and nutrition outcomes is but a part 2 of the puzzle; suggesting that in addition to NL, behaviours are also determined by 3 psychosocial attributes such as self-efficacy [7, 18]. Self-efficacy is as an individual's belief 14 in their capability to perform a specified task aimed at achieving a particular desired outcome [19].

Self-efficacy has powerful influence over an individual's motivation and persistence to overcome barriers in order to achieve the desired health outcome; self-efficacy perceptions predict people's behaviour as these perceptions determine what individuals do with the knowledge and skills that they have [20]. Accordingly, individuals with higher perceived self-efficacy are more motivated to make the effort required to overcome any challenges that might arise, making them more likely to accomplish the desired health outcomes as compared to their counterparts with lower levels of perceived self-efficacy [21]. 
1 Relating to nutrition, previous research has shown that self-efficacy predicts adoption of 2 positive dietary practices such as weight control, increased consumption of fruit and 3 vegetables and utilizing the information on nutrition fact labels on groceries [22-23]. To 4 measure perceived self-efficacy, we must tailor scales to the specific domain of interest [24].

5 In the present study, we applied a recently developed and Rasch-validated 5-item 'Self6 Efficacy in Body and Health' (SEBH) scale. The scale is a valid and reliable measure of 7 adolescents' perceived self-efficacy in the natural science topic 'Body and Health' [17]. The 8 SEBH scale assesses the perceived self-efficacy of adolescents in mastering the health content taught in 'Body and Health' topic in the Norwegian science curriculum (Table 3). 'Body and Health' focuses on the structure of our bodies, how the body changes over time; and the effect of nutrition and lifestyle on the body [25].

\section{Table 3 Stem and wording of items in the SEBH scale ${ }^{a}$}

How do you agree with the claim (1 = Disagree strongly, 6 = Agree strongly $)$ ?

\begin{tabular}{|l|l|}
\hline Item & \multicolumn{1}{|c|}{ Item wording } \\
\hline 1 & $\begin{array}{l}\text { I am confident that if I have to learn something very thoroughly in Body and } \\
\text { Health, I will be able to manage it. }\end{array}$ \\
\hline 2 & $\begin{array}{l}\text { I am confident that I can do an excellent job with difficult tasks in Body and } \\
\text { Health }\end{array}$ \\
\hline 3 & $\begin{array}{l}\text { I am confident that I can do very well in tests in Body and Health. } \\
\text { Health }\end{array}$ \\
\hline 5 & $\begin{array}{l}\text { I am confident that I can apply the knowledge that I have in Body and Health in } \\
\text { new and unfamiliar situations }\end{array}$ \\
\hline
\end{tabular}


1 The structural equation modelling (SEM) framework and study hypotheses

2 We explored the association between SEBH and the two aspects of CNL (CNLEval, 3 CNLEng) by specifying, identifying, estimating and evaluating a simple yet appropriate SEM model [26]. As these three latent traits were measured using six-point rating scale items at the ordinal level, we used the diagonally or variance weighted least square estimator (DWLS) available in the software package Lisrel 9.30.

\section{Steps in conducting a SEM analysis}

Model specification: The model in Figure 1 shows the measurement models of each of the three latent variables or factors and depicts the hypothesized relationships between them when they are allowed to freely covary.

The simple SEM model in Figure 2 specifies that adolescents' perceived self-efficacy in Body and Health (SEBH) explains a 'significant' portion of the variability in the two subdomains CNLEval and CNLEng of CNL. We might say that in our model, SEBH serves as an independent latent variable, and that CNLEval and CNLEng are dependent latent variables.

According to Bandura [21], persons' self-efficacy perceptions largely determine their behavior, as these perceptions determine what they do with the knowledge and skills that they have acquired. For example, a study on adolescents following an intervention study with chefs showed that the students that had higher levels of self-efficacy were more likely to apply the knowledge and skills obtained and participate in food shopping and meal preparation, at home [27].

Consequently, we expect that perceived self-efficacy in a nutrition-related topic such as 'Body and Health' (SEBH) might be associated with a greater willingness to engage (CNLEng) in diet-related practices and or issues. This is modelled as a direct effect between SEBH and CNLEng in Figure 2.

Previous market studies show that perceived self-efficacy determines the extent of elaboration during information processing [28]. For that reason, we expect adolescents who feel that they are competent enough to understand the information they have (high selfefficacy), to engage in a more detailed processing of information (CNLEval) than their counterparts with lower levels of self-efficacy do. This is modelled as a direct effect between SEBH and CNLEval in Figure 2. 
1 Several studies indicate that while adolescents have access to nutrition information, they do not use it because they find it too confusing to understand [12-13]. Other studies show that individuals engage in a more detailed process of information-appraisal in order to sustain or enhance their state of well-being [28-29]. From this standpoint, we expect that the extent to which adolescents engage in dietary related practices depend on how they evaluate nutrition information. Thus, we also hypothesize that how adolescents evaluate nutrition information (CNLEval) affects their level of engagement in dietary practices (CNLEng). In Figure 2 this is modelled as an indirect effect where CNLEval facilitates or "mediates" the relationship between SEBH and CNLEng. In this way, CNLEval is both an independent variable and a mediator variable.

Model identification: This second step in SEM analysis involves determining whether the sample variance-covariance matrix $(s)$ contains enough information or "distinct values" to obtain a unique solution for each model parameter or "free parameter" to be estimated. These parameters are typically item factor loadings, unique error variances of the items (an item error term consisting of "item specific true variance" and "measurement error"), independent latent variables, dependent latent variable residuals (each dependent latent variable has a prediction error term), and structure coefficients.

The number of distinct values (DV) in the sample matrix $s$ - variances on the diagonal and covariances off-diagonal - as $p(p+1) / 2=12(12+1) / 2=78$, where $p=12$ is the total number of items or indicators in the SEM. The number of free parameters (FP) to be estimated in the apriori specified SEM were counted as follows; 9 factor loadings (with 3 other factor loadings fixed to 1), 12 item unique variances, 1 independent latent variable (SEBH), 2 dependent latent variable residuals ( 1 for CNLEval and 1 for CNLEng) and 3 structure coefficients. With 27 FP to be estimated in our model and 78 DV in the sample matrix, the apriori specified SEM is 'over-specified' with 78-27 = 51 degrees of freedom $(d f)$. When DV $>$ FP the order condition is fulfilled and "goodness-of-fit" (GOF) indicators can be estimated in addition to FP.

The third step in SEM analysis, model estimation, aims at estimating a numerical value for each FP.

The objective of model estimation is to obtain a model-based (implied) polychoric variancecovariance matrix $(\Sigma)$ with elements as close as possible to the elements in the sample (data) 
1 covariance matrix (S) [30-31]. As all items or indicators are rating scale items at the ordinal

2 level, we estimated the FP by using diagonally weighted least squares (DWLS) estimator.

3 DWLS is an asymptotic distribution free (ADF) estimator that does not assume any underlying normal distribution of the data. However, to compare the values of goodness-of-fit indexes (I) to GOFI "target values" obtained in simulation studies, we estimated GOF indexes using maximum likelihood (ML) estimator.

The fourth step, model evaluation, refers to evaluating the discrepancy between $S$ and $\Sigma$ through assessing fit. The closer the model-implied covariance matrix is to the sample (or data) covariance matrix, the better the model fit. As there is no 'gold standard' of the GOFI, we examine and report model fit against different GOFI. The most widely reported GOFI is the chi-square test $\left(\chi^{2}\right)$; statistically significant values suggest imperfect model fit, and that the model be rejected. However, because the $\chi^{2}$ test is sensitive to sample size, and assumes underlying multivariate normal distribution, it is advisable to report at least one index from each of the three classes of GOFI, namely, the absolute, parsimony-adjusted, and comparative).

Absolute GOF indexes depict how well the specified model explains the proportions of the covariances in the sample data matrix and measure the degree to which the hypothesized model as a whole is consistent with the empirical data. Examples of these include the SatorraBentler scaled chi-square $\left(\chi^{2}\right)$, the reduced chi-square $\left(\chi^{2} / d f\right)$, and the standardized root-meansquare residual (SRMR).

SB $\left(\chi^{2}\right)$ values $>0.05$ point to a good model fit; $\left(\chi^{2} / d f\right)$ values <3 suggest good fit; SRMR values < 0.05 suggest a well-fitting model, a value of 0.00 indicates 'perfect' fit; however, values as high as 0.08 also point to 'acceptable' model fit [33-34].

Parsimony-adjusted GOF indexes are concerned with model parsimony; they have an 'inbuilt correction' for model complexity, thereby favouring simpler models (those with fewer parameters) [35].

An example is the root mean square error of approximation (RMSEA) with its associated "close" fit (C-fit) value at a 90\% confidence interval; this measures the discrepancy between the observed and model implied matrices but penalises non-parsimony models containing many parameters i.e., models with less degrees of freedom as the difference between DV and 
1 FP is smaller. RMSEA values <0.06 suggest good model fit, values $0.08-0.10$ suggest 2 mediocre fit, while values $>0.10$ point to poor fit; C-Fit values $>0.05$ suggest acceptable 3 model fit [36].

Finally, incremental or comparative fit GOF indexes, like the comparative fit index (CFI) and the non-normed fit index (NNFI) also known as the Tucker-Lewis index (TLI), assess fit relative to a baseline model hypothesizing no relationships among the variables and are therefore rather liberal CFI value of $\geq 0.95$ suggest good model fit; NNFI values $>0.95$ points to good model fit [37].

Whereas the aforementioned fit indexes give an overall view of model fit, it is important to evaluate the three measurement models separately (Figure 1). To do this, we examine the factor loadings; the latent variable should explain at least $50 \%$ of the common variance in each item or indicator, each factor loading should exceed 0.70 i.e., $0,71^{2}=0.50$. As all scales were validated using Rasch-modelling prior to the SEM analysis, we expect that all factor loadings exceed 0.70 by a good margin.

In addition to the global GOF indexes, we also evaluate SEM models by using local indices; insignificant residual matrix elements (values exceeding 2.56) indicate substantial specification and prediction error.

\section{Sampling and data collection}

We randomly selected 200 schools from the list of lower secondary schools in Norway and the respective school principals were contacted via email and telephone, seeking consent to volunteer in the study. Of these, 58 (approx. 30\%) schools accepted and were included in the study. During the period of April to May 2015, we collected data from 1622 tenth grade students aged 15-16 years who responded by using an electronic survey system.

\section{Results}

Of the three scales, CNLEval had the highest average number of missing responses for its five items (97.6), whereas the SEBH scale and the CNLEng scale items on average had 61.2 and 62 missing responses, respectively.

\section{(Insert Table 4 here)}


1 Table 4 shows the estimates of the 27 free parameters for the SEM model in Figure 2. We may say that all items are valid indicators as all factor loadings exceed 0.70 i.e., the respective latent factor or latent trait "governs the responses to the items" and can explain more than $0.70^{2}$ or $50 \%$ of the variance in the responses to the item. Further, this means that less than $50 \%$ of the variance is "unique" or unexplained by the respective factor (therefore all the 12 unique variances in Table 4 is smaller than 0.5 ).

By comparing the model fitted variance-covariance matrix (the "model implied matrix" containing the variances and covariances based upon the model) to the sample variancecovariance matrix $s$, we form the residual matrix. The standardized residual matrix show that all but four elements were smaller than $z=2.56$, meaning that there are small differences between the elements of the sample variance-covariance matrix and the model fitted or model implied variance-covariance matrix. This indicates that our specified SEM-model describes the patterns in the observed data sufficiently well.

\section{(FIGURE 1 here)}

Figure 1: The SEM in which the three measurement models for the latent variables freely covary.

We applied robust diagonally weighted least square (DWLS) estimation as rating scale data are categorical. Latent variables are drawn as ellipses, whereas the rectangles represent the observed variables or items. The arrows pointing towards the rectangles represent the unique variances of each item.

\section{(FIGURE 2 here)}

Figure 2: Path diagram of the SEM hypothesizing that SEBH directly influences CNLEval, CNLEng, indirectly influences CNLEng through CNLEval

Owing to rating scale items (ordinal variables), we used polychoric variance-covariance matrix and robust diagonally weighted least squares (DWLS) estimation. Using standardized solution with reference variables (one factor loading fixed to 1 for each measurement model), the polychoric correlation between the latent factor and the respective item can be estimated as sqrt(1- unique error variance). Latent variables are drawn as ellipses, whereas the rectangles represent the observed variables or items. 
1 All three structure coefficients in Figure 2 are significantly different from zero: The structure 2 coefficient or parameter estimate linking SEBH to CNLEval has a z-distributed Wald statistic $3=\left(B-B_{0}\right) / \mathrm{SE}=(.532-.000) / .025=21.28>2.56$, with two-tailed $p$-value $>.01$, the structure 4 coefficient or parameter estimate linking SEBH to CNLEng has a z-distributed Wald statistic $5=(.309-.000) / .034=9.088>2.56$, with two-tailed $p$-value $>.01)$, and the structure 6 coefficient linking CNLEv and CNLEng $=(.282-.000) / .038=7.421>2.56$, with two-tailed $7 p$-value $>.01$.

8 The overall predictive validity of the structural model was acceptable: Using the structural 9 coefficients found in the unstandardized and standardized LISREL beta and gamma output matrices, where the standardized structural coefficients are also available in Figure 2, we estimated the total effects. We found that SEBH acts as a substantial "predictor" of students' CNLEval (unstandardized total effect $=$ unstandardized direct effect $=.532$; standardized total effect $=$ standardized direct effect $=.552$ ) and of students' CNLEng (unstandardized total effect $=$ unstandardized direct effect + unstandardized indirect effect $=.309+(.532 \mathrm{x}$ $.282)=.309+.150=.459 ;$ standardized total effect $=.319+(.552 \times .281)=.319+.155=$ .475), where the unstandardized total effect .459 is significantly different from zero (zdistributed Wald statistic $=.459-.000 / .027=17.1>2.56$ with two-tailed $p$-value $>.01)$. The standard error .027 associated with the total effect .459 was available in the LISREL "Total Effects of KSI on ETA" output matrix.

According to GOF index target values, the specified SEM model seems to sufficiently describe the structure of the sample data (Table 5). 
Table 5: Model evaluation by goodness-of-fit (GOF) indexes.

\begin{tabular}{|c|c|c|c|c|c|c|c|}
\hline \multirow[t]{2}{*}{ Model } & \multicolumn{3}{|c|}{ Absolute GOF indexes } & \multicolumn{2}{|c|}{$\begin{array}{l}\text { Parsimony-adjusted } \\
\text { GOF indexes }\end{array}$} & \multicolumn{2}{|c|}{$\begin{array}{l}\text { Incremental } \\
\text { GOF indexes }\end{array}$} \\
\hline & $\begin{array}{l}\text { SB-scaled } \\
\chi^{2}\end{array}$ & $\begin{array}{l}\text { Reduced } \\
\text { chi-square } \\
\chi^{2} / d f\end{array}$ & SRMR & $\begin{array}{l}\text { RMSEA } \\
(90 \% \mathrm{CI})\end{array}$ & Cfit & CFI & NNFI \\
\hline $\begin{array}{l}\text { Model in Figure } \\
1(d f=51, N= \\
1453)\end{array}$ & $\begin{array}{l}164.543 \\
p=\mathbf{0 . 0 0 0}\end{array}$ & 3.226 & 0.025 & $\begin{array}{l}0.067 \text { (0.061; } \\
0.073)\end{array}$ & 0.000 & 0.991 & 0.989 \\
\hline $\begin{array}{l}\text { Model in Figure } \\
2(d f=51, N= \\
1453)\end{array}$ & $\begin{array}{l}158.765 \\
p=\mathbf{0 . 0 0 0}\end{array}$ & 3.113 & 0.027 & $\begin{array}{l}0.065(0.059 ; \\
0.071)\end{array}$ & $\mathbf{0 . 0 0 0}$ & 0.977 & 0.970 \\
\hline Target value & $p>.05$ & $<3$ & $<.05$ & $\begin{array}{l}<.06(<.05 ; \\
<.08)\end{array}$ & $>.05$ & $>.95$ & $>.95$ \\
\hline
\end{tabular}

${ }^{*}$ GOF index values reported are based on model estimation by using robust ML estimator.

$2 d f=$ degrees of freedom; SB-scaled $\chi^{2}=$ Satorra-Bentler scaled chi-square,

3 SRMR=Standardized Root Mean Square Residual, RMSEA=Root Mean Square Error of

4 Approximation, $\mathrm{CFit}=p$-value for test of close fit, $\mathrm{CFI}=\mathrm{Comparative}$ Fit Index, NNFI=Non-

$5 \quad$ Normed Fit Index.

$6 \quad *$ Bold values indicate mediocre to poor data-model fit.

7

8

9

\section{Discussion}


1 Empirical findings supported the hypothesis that self-efficacy in a science subject was associated with critical nutrition literacy (CNL). This significant positive association is similar to findings from a study conducted on young adolescents in Norway in which students that expected to perform well on the science test reported higher levels of engagement in dietary behaviours than their counterparts [7].

Similarly, consumer research shows that for individuals that are concerned about their health, the extent to which they engage in actions that promote their health depends on their nutrition self-efficacy [28]. 'Nutrition self-efficacy' refers to a person's belief in his or her ability to overcome the barriers that are associated with healthful eating and is often associated with healthy dietary behaviour [28].

The extent to which young adolescents undertake positive dietary behaviours depends on their perceptions of competency to accomplish the task (self-efficacy) and understanding of the information relating to the task. Similarly, Mai \& Hoffmann suggest that nutrition selfefficacy, which in turn influences the extent of elaboration in information processing, determines food decision strategies [28]. While there is no obvious directional association, findings in the present study support this notion, as shown by the stronger relationship between self-efficacy and CNLEval in comparison to that between SEBH and CNLEng, and CNLEval and CNLEng.

It is for this reason that studies exploring the level of engagement in positive practices such as using nutrition labels during shopping suggest a two-tiered approach to increasing adolescents' use of nutrition labels through enhancing adolescents' confidence in understanding nutrition labels and simplifying the information on the nutrition labels [29].

Compared to self-efficacy, there are fewer measures CNL; the present study showed that selfefficacy is a strong predicting factor for CNL. Thus, in the absence of instruments that specifically measure CNL, it is possible to use existing measures of self-efficacy during screening to forecast individual's possible outcomes in nutrition interventions.

The extent to which adolescents are engaged in participating in daily dietary-related practices is important as it may influence their food consumption decisions, self-efficacy and development of the skills required to prepare 'healthier' foods. This justification finds support in an intervention study whereby children that closely participated in food 
1 preparation reported an increased interest for more opportunities to practice the new food 2 preparation skills and an increase in the consumption of vegetables [27].

3

4 Whereas the present study showed a significant direct effect of self-efficacy on the two 5 domains of CNL, this finding differs from previous studies in which engagement in 6 household food tasks contributed to increased self-efficacy [38-39]. They argue that 7 perceived self-efficacy is greater when individuals have practical experience with the 8 necessary skills for completion. This exhibits the interconnected nature of psychosocial attributes and the skills associated with the critical domain of nutrition literacy, a notion that is consistent with Nutbeam's description of the skills associated with the critical level of health literacy namely higher-level cognitive and interactive social skills [3]. Consequently, when planning for and evaluating the outcome of health or nutrition programs, it is important to consider psychosocial attributes such as self-efficacy and motivation.

\section{Conclusions}

The present study exhibited the relationship between self-efficacy and the two subdomains of critical nutrition literacy- CNLEval and CNLEng, basing on a theoretically founded structural equation model. Self-efficacy was directly related to 'engagement in dietary behaviour' and indirectly to 'evaluation of nutrition information' via 'engagement in dietary behaviour'. This gives insight into the interaction of psychosocial attributes particularly self-efficacy and critical nutrition literacy in adolescents. The findings can inform the development of policies, interventions addressing critical nutrition literacy needs of adolescents within the larger scope of media use and educational settings. 


\section{Declarations}

\section{Ethics approval and consent to participate}

3 No ethics approval was required. Data analysed was collected as part of a field test trial of the

4 national science test.

\section{Consent for publication}

$6 \quad$ Not applicable

\section{$7 \quad$ Availability of data and materials}

8 The data that support the findings of this study are available from The Norwegian Directory

9 for Education but restrictions apply to the availability of these data, which were used under

10 license for the current study, and so are not publicly available. Data are however available

11 from the authors upon reasonable request and with permission of The Norwegian Directory

12 for Education.

\section{Competing interests}

14 The authors declare that they have no competing interests

15 Funding

16 Not applicable. This study did not receive a specific grant or funding.

\section{Author contributions}

DAN and ØG conducted the statistical analysis and drafted the manuscript.

KSP developed the questionnaire items applied in the study and offered guidance on the contents of the manuscript.

21 SH read through and contributed towards the contents of the manuscript.

$22 \varnothing \mathrm{G}$ conceived the study, and managed the data collection.

23 All authors read and approved the final manuscript.

\section{Acknowledgements}

25 The authors would like to thank the students who responded and the extraordinary support from school principals and teachers who selflessly facilitated the data collection process.

27 We also would like to thank Lena Victoria Nordheim for contributing to the development of 28 the CNLEval scale items. 
2 1. Kickbusch I. Health literacy: engaging in a political debate. Int J Publ Health Sci. $32009 ; 54(3): 131-2$.

4 2. Silk KJ, Sherry J, Winn B, Keesecker N, Horodynski MA, Sayir A. Increasing 5 nutrition literacy: testing the effectiveness of print, web site, and game modalities. J Nut Educ 6 Behav. 2008;40(1):3-10.

73 . Nutbeam D. Health literacy as a public health goal: a challenge for contemporary 8 health education and communication strategies into the 21 st century. Health Promot Int. 9 2000;15(3):259-67.

4. Velardo S. The nuances of health literacy, nutrition literacy, and food literacy. J Nut Educ Behav. 2015;47(4):385-9. e1.

5. Pettersen KS. Health literacy og Nutrition literacy. In: Dagrun E, Torheim LE, Øverby NC, editors. Samfunnsernæring. Oslo: Universitetsforlaget; 2019. p. 167 - 74.

6. Guttersrud $\varnothing$, Dalane JØ, Pettersen S. Improving measurement in nutrition literacy research using Rasch modelling: examining construct validity of stage-specific 'critical nutrition literacy' scales. Public Health Nutr. 2014;17(4):877-83.

7. Guttersrud $\varnothing$, Pettersen KS. Young adolescents' engagement in dietary behaviour-the impact of gender, socio-economic status, self-efficacy and scientific literacy. Methodological aspects of constructing measures in nutrition literacy research using the Rasch model. Public Health Nutr. 2015;18(14):2565-74.

8. Modell, J., \& Goodman, M. (1990). Historical perspectives. In S. S. Feldman \& G. R. Elliott (Eds.), At the threshold: The developing adolescent (pp. 93-122). Cambridge, MA: Harvard University Press.

9. Velardo, S., \& Drummond, M. (2016). Emphasizing the child in child health literacy research. J Child Health Care 21(1), 5-13.doi:https://doi.org/10.1177/1367493516643423

10. Talagala, I. A., \& Arambepola, C. (2016). Use of food labels by adolescents to make healthier choices on snacks: a cross-sectional study from Sri Lanka. BMC Public Health 16(1), 739. doi:10.1186/s12889-016-3422-1

11. Dong, Z. (2015). How to persuade adolescents to use nutrition labels: effects of health consciousness, argument quality, and source credibility. Asian J Commun. 25(1), 84-101. doi:10.1080/01292986.2014.989241 
12. Saha, S., Vemula, S. R., Mendu, V. V. R., \& Gavaravarapu, S. M. (2013). Knowledge and practices of using food label information among adolescents attending schools in $\begin{array}{llllll}\text { Kolkata, } & \text { India. } & \mathrm{J} & \text { Nut } & \text { Educ } & \text { Behav }\end{array}$ doi:http://dx.doi.org/10.1016/j.jneb.2013.07.011

13. Wojcicki, J. M., \& Heyman, M. B. (2012). Adolescent nutritional awareness and use of food labels: Results from the national nutrition health and examination survey. BMC Pediatr, 12(1), 55. doi:https://doi.org/10.1186/1471-2431-12-55

14. Krause, C., Sommerhalder, K., \& Beer-Borst, S. (2016). Nutrition-specific health literacy: development and testing of a multi-dimensional questionnaire. Ernahrungs Umschau, 63(11), 214-220. doi:10.4455/eu.2016.046

15. Nguyen, T. H., Paasche-Orlow, M. K., Kim, M. T., Han, H.-R., \& Chan, K. S. (2015). Modern measurement approaches to health literacy scale development and refinement: overview, current uses, and next steps. J Health Commun. 20(sup2), 112-115. doi:10.1080/10810730.2015.1073408

16. Naigaga DA, Pettersen KS, Henjum S, Guttersrud $\varnothing$. Assessing adolescents' perceived proficiency in critically evaluating nutrition information. Int J Behav Nutr Phys Act. 2018;15(1):61.

17. Naigaga, Pettersen, Henjum, Guttersrud. Assessing adolescent self-efficacy in Body and Health - Exploring the psychometric properties of the SEBH scale. NORDINA. 2019.

18. Guntzviller, L. M., King, A. J., Jensen, J. D., \& Davis, L. A. (2017). Self-efficacy, health literacy, and nutrition and exercise behaviors in a low-income, Hispanic population. $\mathbf{J}$ Immigr Minor Health 19(2), 489-493. doi:https://doi.org/10.1007/s10903-016-0384-4 19. Bandura, A. (1977). Self-efficacy: toward a unifying theory of behavioral change. Psychol Rev. 84(2), 191. doi:http://psycnet.apa.org/doi/10.1037/0033-295X.84.2.191

20. Anderson, E. S., Winett, R. A., \& Wojcik, J. R. (2000). Social-cognitive determinants of nutrition behavior among supermarket food shoppers: A structural equation analysis. Health Psychol. 19(5), 479. doi:http://psycnet.apa.org/doi/10.1037/0278-6133.19.5.479 21. Bandura, A. (1989). Human agency in social cognitive theory. Am Psychol. 44(9), 1175. doi:https://doi.org/10.1037/0003-066X.44.9.1175

22. Bandura, A. (1997). Editorial: The anatomy of stages of change. Am J Health Promot. 12(1), 8-10. doi:https://doi.org/10.4278/0890-1171-12.1.8

23. Aboulnasr K. The role of self-efficacy, response efficacy, and nutrition knowledge in consumers' utilization of nutrition labels. Mark Manag. 2013; 2. 
1 24. Bandura A. Guide for constructing self-efficacy scales. Self-efficacy beliefs of

2 adolescents. 5. Connecticut, USA: Information Age Publishing; 2006. p. 307-37.

3 25. KD. Fag - Fordypning - Forståelse. En fornyelse av Kunnskapsløftet. In: Minister 4 TOOTP, editor. 2016.

5 26. Lomax RG, Schumacker RE. A beginner's guide to structural equation modeling:

6 psychology press; 2004

7 27. Caraher M, Seeley A, Wu M, Lloyd S. When chefs adopt a school? An evaluation of a 8 cooking intervention in English primary schools. Appetite. 2013;62:50-9.

9 28. Mai R, Hoffmann S. Taste lovers versus nutrition fact seekers: how health consciousness and self- efficacy determine the way consumers choose food products. J Consumer Behav. 2012;11(4):316-28.

29. Petty, R. E. \& Cacioppo, J. T., (1986). The elaboration likelihood model of persuasion. In L. Berkowitz (Ed.), Advances in Experimental Social Psychology (pp. 123205). New York: Academic Press.

30. Wang Q, Oostindjer M, Amdam GV, Egelandsdal B. Snacks with nutrition labels: tastiness perception, healthiness perception, and willingness to pay by Norwegian adolescents. Journal of nutrition education and behavior. 2016;48(2):104-11. e1.

31. Rossman M. Involving children in household tasks: is it worth the effort. Research Works, University of Minnesota College of Education and Human Development Retrieved December. 2002;21:2004.

32. Hill, L., Casswell, S., Maskill, C., Jones, S., \& Wyllie, A. (1998). Fruit and vegetables as adolescent food choices in New Zealand. Health promotion international, 13(1), 55-65. 
Figures

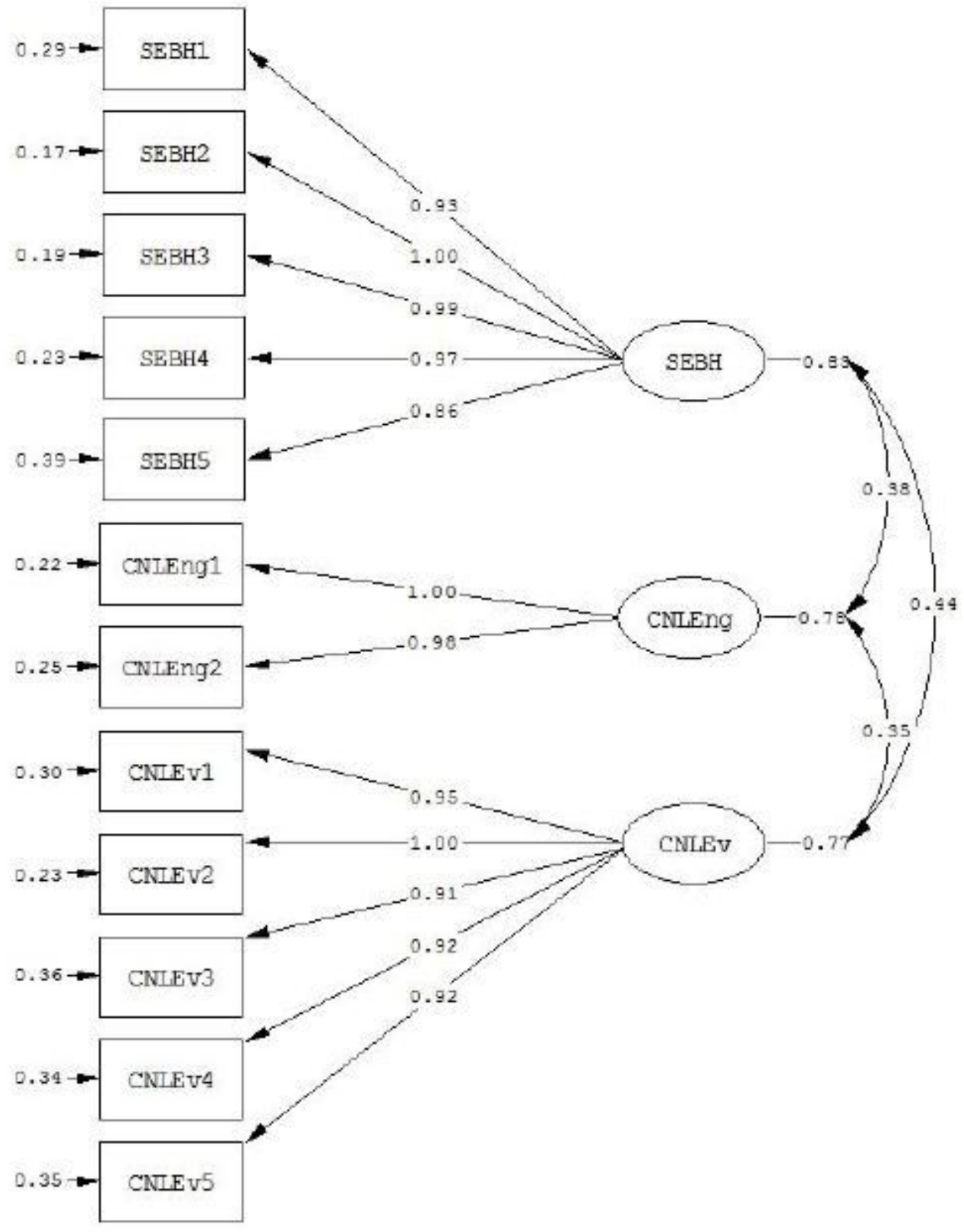

Figure 1

The SEM in which the three measurement models for the latent variables freely covary. 


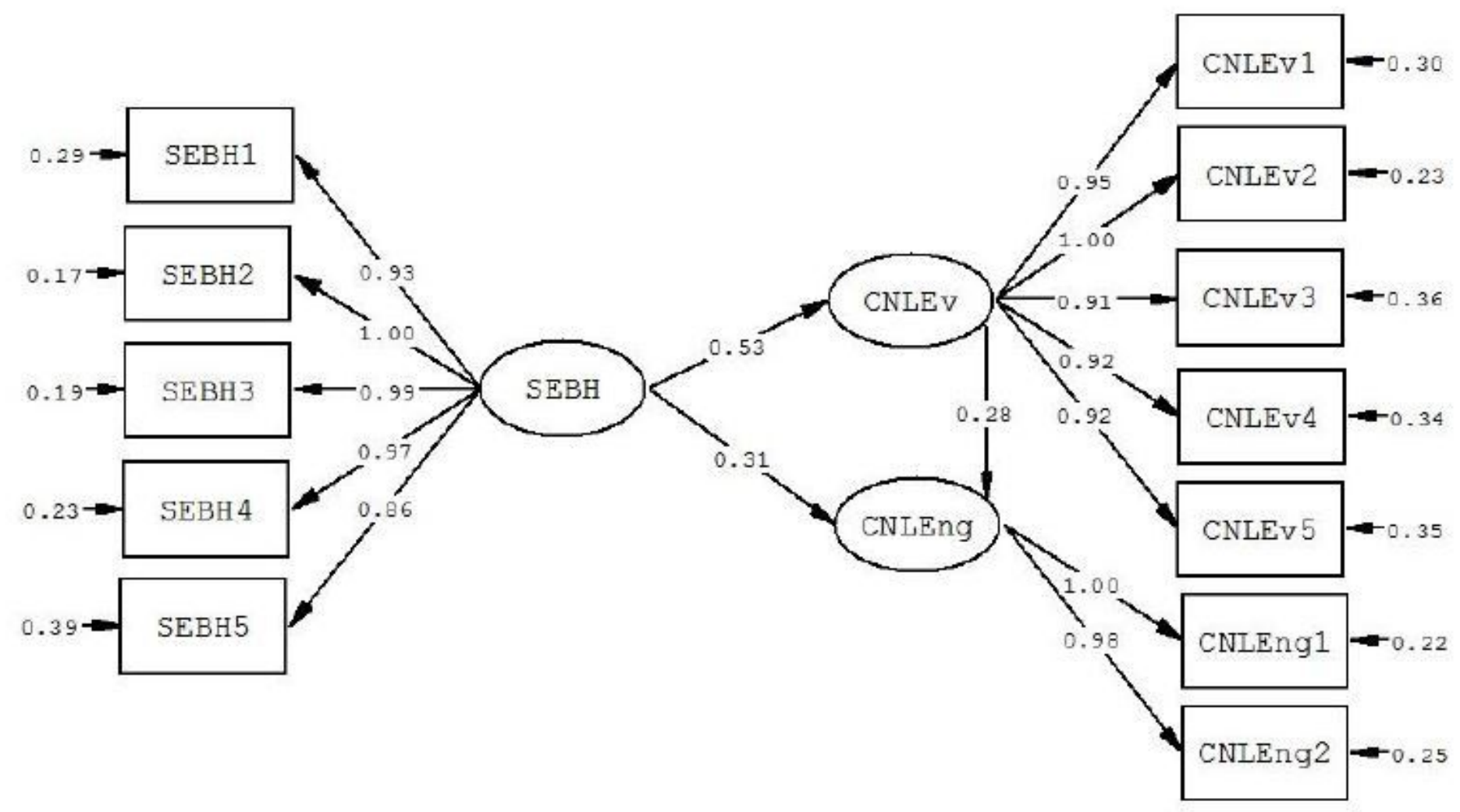

Figure 2

Path diagram of the SEM hypothesizing that SEBH directly influences CNLEval, CNLEng, indirectly influences CNLEng through CNLEval

\section{Supplementary Files}

This is a list of supplementary files associated with this preprint. Click to download.

- RelatingaspectsofCNLTable4.pdf 